\title{
Natural Killer T-Cell
}

National Cancer Institute

\section{Source}

National Cancer Institute. Natural Killer T-Cell. NCI Thesaurus. Code C129906.

A subset of T-lymphocytes that coexpress an alpha/beta T-cell receptor, and variety of molecular markers that are typically associated with natural killer (NK) cells, which may include CD161, CD16, CD68, and granzyme. NK T-cells recognize glycolipids presented by antigen-presenting glycoprotein CD1d on antigen presenting cells; antigen recog nition stimulates a rapid release of various cytokines from the NK T-cells. 